\title{
Strong Convergence Theorems for a Common Fixed Point of a Family of Asymptotically $k$-Strict Pseudocontractive Mappings
}

\author{
H. Zegeye ${ }^{1}$ and N. Shahzad ${ }^{2}$ \\ ${ }^{1}$ Department of Mathematics, University of Botswana, Private Bag 00704, Gaborone, Botswana \\ ${ }^{2}$ Department of Mathematics, King Abdulaziz University, P.O. Box 80203, Jeddah 21589, Saudi Arabia
}

Correspondence should be addressed to N. Shahzad; nshahzad@kau.edu.sa

Received 21 September 2012; Accepted 11 December 2012

Academic Editor: Cristina Marcelli

Copyright (c) $2013 \mathrm{H}$. Zegeye and N. Shahzad. This is an open access article distributed under the Creative Commons Attribution License, which permits unrestricted use, distribution, and reproduction in any medium, provided the original work is properly cited.

We provide an iterative process which converges strongly to a common fixed point of finite family of asymptotically $k$-strict pseudocontractive mappings in Banach spaces. Our theorems improve and unify most of the results that have been proved for this important class of nonlinear operators.

\section{Introduction}

Let $E$ be a real normed linear space with dual $E^{*}$. A gauge function $\varphi:[0, \infty]:=R^{+} \rightarrow R^{+}$is a continuous and strictly increasing function satisfying $\varphi(0)=0$ and $\varphi(t) \rightarrow \infty$, as $t \rightarrow \infty$. The generalized duality mapping from $E$ to $2^{E^{*}}$ associated with the gauge function $\varphi$ (see, e.g., [1]) is defined by

$$
\begin{gathered}
J_{\varphi}(x):=\left\{x^{*} \in E^{*}:\left\langle x, x^{*}\right\rangle=\|x\| \varphi(\|x\|),\right. \\
\left.\left\|x^{*}\right\|=\varphi(\|x\|)\right\}, \quad \forall x \in E,
\end{gathered}
$$

where $\langle\cdot, \cdot\rangle$ denotes the duality pairing. In the case that $\varphi(t)=$ $t$, the duality mapping $J_{\varphi}=J$ is called the normalized duality mapping.

Following Browder [2], we say that a Banach space $E$ has a weakly continuous duality mapping if there exists a gauge $\varphi$ for which the duality mapping $J_{\varphi}$ is single valued and weakto-weak ${ }^{*}$ sequentially continuous (i.e., if $\left\{x_{n}\right\}$ is a sequence in $E$ weakly convergent to a point $x$, then the sequence $J_{\varphi}\left(x_{n}\right)$ converges weak ${ }^{*}$ to $J_{\varphi}(x)$ ). It is known that $l_{p}$ has a weakly continuous duality mapping with a gauge function $\varphi(t)=$ $t^{p-2}$, for all $1<p<\infty$.

Let $K$ be a nonempty subset of $E$. A mapping $T: K \rightarrow$ $K$ is called asymptotically $k$-strict pseudocontractive, with sequence $\left\{l_{n}\right\} \subseteq[1, \infty), \lim _{n \rightarrow \infty} l_{n}=1$ (see, e.g., [3-6]) if for all $x, y \in K$, there exist $j(x-y) \in J(x-y)$ and a constant $k \in[0,1)$ such that

$$
\begin{aligned}
& \left\langle T^{n} x-T^{n} y, j(x-y)\right\rangle \\
& \quad \leq l_{n}\|x-y\|^{2}-k\left\|\left(I-T^{n}\right) x-\left(I-T^{n}\right) y\right\|^{2},
\end{aligned}
$$

for all $n \geq 1$.

If $I$ denotes the identity operator, then (2) can be equivalently written as

$$
\begin{aligned}
& \left\langle\left(I-T^{n}\right) x-\left(I-T^{n}\right) y, j(x-y)\right\rangle \\
& \quad \geq k\left\|\left(I-T^{n}\right) x-\left(I-T^{n}\right) y\right\|^{2}-\left(l_{n}-1\right)\|x-y\|^{2},
\end{aligned}
$$

for all $n \geq 1$.

If $E=H$, a real Hilbert space, it is shown by Osilike et al. [4] that (2) (and hence (3)) is equivalent to the inequality

$$
\begin{aligned}
\left\|T^{n} x-T^{n} y\right\|^{2} \leq & \left(1+2\left(l_{n}-1\right)\right)\|x-y\|^{2} \\
& +\lambda\left\|\left(I-T^{n}\right) x-\left(I-T^{n}\right) y\right\|^{2},
\end{aligned}
$$

where $\lambda=(1-2 k) . T$ is called uniformly Lipschitz if there exists $L \geq 0$ such that $\left\|T^{n} x-T^{n} y\right\| \leq L\|x-y\|$ for all $x, y \in D(T)$. It is shown in [4] that an asymptotically $k$-strict pseudocontractive mapping is uniformly Lipschitz.

The class of asymptotically $k$-strict pseudocontractive mappings was first introduced in Hilbert spaces by Liu [5] He proved the following theorem. 
Theorem $\mathbf{Q}$ (see [5]). Let $K$ be a closed convex and bounded subset of a Hilbert space $H$. Let $T: K \rightarrow K$ be completely continuous asymptotically $k$-strict pseudocontractive mapping for some $0 \leq k<1$ with sequence $\left\{l_{n}\right\} \subset[0, \infty)$ such that $\sum\left(l_{n}-1\right)<\infty$ and $F(T) \neq \emptyset$. Let $\left\{x_{n}\right\}$ be a sequence generated by the modified Mann's iteration method:

$$
x_{n+1}=\alpha_{n} x_{n}+\left(1-\alpha_{n}\right) T^{n} x_{n}, \quad n \geq 1,
$$

where $\left\{\alpha_{n}\right\}$ is a real sequence satisfying $\epsilon \leq \alpha_{n} \leq 1-k-\epsilon$ for all $n \geq 1$ and some $\epsilon>0$. Then, $\left\{x_{n}\right\}$ converges strongly to a fixed point of $T$.

The iteration scheme (5) is called modified Mann's iterative processes which was introduced by Schu $[7,8]$ and has been used by several authors (see, e.g., [3-5, 9-17]). We observe that Liu [5] proved strong convergence of scheme (5) to a fixed point of asymptotically $k$-strict pseudocontractive mapping $T$ with additional assumption that $T$ is completely continuous, where $T: C \rightarrow C$ is said to be completely continuous if for every bounded sequence $\left\{x_{n}\right\}$, there exists a subsequence, say $\left\{x_{n_{j}}\right\}$ of $\left\{x_{n}\right\}$ such that the sequence $\left\{T x_{n_{j}}\right\}$ converges strongly to some element of the range of $T$.

In [12], Kim and $\mathrm{Xu}$ studied weak convergence theorem for the class of asymptotically $k$-strict pseudocontractive mappings in the frame work of Hilbert spaces. In fact, they proved the following.

Theorem KX (see [12]). Let $K$ be a closed and convex subset of a Hilbert space $H$. Let $T: K \rightarrow K$ be an asymptotically $k$-strict pseudocontractive mapping for some $0 \leq k<1$ with sequence $\left\{l_{n}\right\} \subset[0, \infty)$ such that $\sum\left(l_{n}-1\right)<\infty$ and $F(T) \neq \emptyset$. Let $\left\{x_{n}\right\}$ be a sequence generated by the modified Mann's iteration method:

$$
x_{n+1}=\alpha_{n} x_{n}+\left(1-\alpha_{n}\right) T^{n} x_{n}, \quad n \geq 1,
$$

where $\left\{\alpha_{n}\right\}$ is a real sequence satisfying $k+\lambda \leq \alpha_{n} \leq 1-\lambda$, for all $n \geq 1$, and $\lambda \in(0,1)$. Then, $\left\{x_{n}\right\}$ converges weakly to a fixed point of $T$.

In 2007, Osilike et al. [13] extended Theorem KX by proving weak convergence of scheme (6) to a fixed point of $T$ in the frame work of $q$ uniformly smooth Banach spaces which are also uniformly convex under suitable control conditions.

In 2011, Zhang and Xie [17] extended Theorem of Osilike et al. [13] to a more general real uniformly convex Banach space $E$ with Fréchet differentiable norm. In addition, they proved strong convergence of scheme (5) to a fixed point of asymptotically $k$-strict pseudocontractive mapping provided that $\liminf _{n \rightarrow \infty} d\left(x_{n}, F(T)\right)=0$, where $d\left(x_{n}, F(T)\right)=$ $\inf _{p \in F(T)}\left\|x_{n}-p\right\|$.

However, we observe that the convergence obtained above is either weak or requiring additional assumption like $\liminf _{n \rightarrow \infty} d\left(x_{n}, F(T)\right)=0$ or $T$ is completely continuous. But the requirement that $\liminf _{n \rightarrow \infty} d\left(x_{n}, F(T)\right)=0$ is not easy to verify, as $F(T)$ is in general unknown, and there is also an example of asymptotically $k$-strict pseudocontractive mapping which is not completely continuous as shown below.

An example of asymptotically $k$-strict pseudocontractive mapping which is not completely continuous.
Example 1. Let $E=l_{2}=\left\{\bar{x}=\left\{x_{i}\right\}_{i=1}^{\infty}, x_{i} \in \mathbb{R}, \sum_{i=1}^{\infty}\left|x_{i}\right|^{2}<\right.$ $\infty\}$ and $\bar{B}=\left\{\bar{x} \in l_{2}:\|\bar{x}\| \leq 1\right\}$. Define $T: \bar{B} \rightarrow$ $\bar{B}$ by $T \bar{x}=\left(0, x_{1}^{2}, a_{2} x_{2}, a_{3} x_{3}, \ldots\right)$, where $\left\{a_{k}\right\}_{k=1}^{\infty}$ is a real sequence satisfying $0<a_{k}<1, k \geq 2$, and $\prod_{k=2}^{\infty} a_{k}=$ $1 / 2$. Then it is shown in [13] that $T$ is asymptotically $k$-strict pseudocontractive mapping.

Now, we show that $T$ is not completely continuous. Let $\left\{x_{n}\right\}$ be a sequence in $\bar{B}$ defined by $x_{1}=(1,0,0, \ldots), x_{2}=$ $(0,1,0,0, \ldots), x_{3}=(0,0,1,0,0, \ldots), \ldots$ Then $\left\{x_{n}\right\} \subset \bar{B}$ and $\left\{T x_{n}\right\}=\left\{y_{n}\right\}$ is given by $y_{1}=(0,1,0,0, \ldots), y_{2}=$ $\left(0,0, a_{2}, 0,0, \ldots\right), y_{3}=\left(0,0,0, a_{3}, 0,0, \ldots\right), \ldots$. Hence, since $a_{k} \rightarrow 1$, as $k \rightarrow \infty$, there is no subsequence $\left\{x_{n_{i}}\right\}$ of $\left\{x_{n}\right\}$ such that $\left\{T x_{n_{i}}\right\}$ converges strongly to a point in $\bar{B}$, as $\left\|T x_{n_{i}}-T x_{n_{j}}\right\|=\left\|y_{n_{i}}-y_{n_{j}}\right\|=\sqrt{\left|a_{n_{i}}\right|^{2}+\left|a_{n_{j}}\right|^{2}} \nrightarrow 0$, as $i, j \rightarrow \infty$. Therefore, $T$ is not completely continuous.

Thus, one question is raised naturally: can we obtain a scheme that converges strongly to a fixed point of asymptotically $k$-strict pseudocontractive mappings without those additional assumptions?

It is our purpose in this paper to provide an iterative scheme $\left\{x_{n}\right\}$ which converges strongly to a common fixed point of finite family of asymptotically $k$-strict pseudocontractive mappings in Banach spaces. The assumption that $\liminf _{n \rightarrow \infty} d\left(x_{n}, F(T)\right)=0$ or $T$ is completely continuous is not required.

\section{Preliminaries}

We need the following definitions from [18]. The Banach space $E$ is said to be uniformly convex if, given $\varepsilon>0$, there exists $\delta>0$, such that, for all $x, y \in E$ with $\|x\| \leq 1,\|y\| \leq 1$ and $\|x-y\| \geq \varepsilon,\|(1 / 2)(x+y)\| \leq 1-\delta$. It is well known that $L_{p}, \ell_{p}$, and Sobolev spaces $W_{m}^{p},(1<p<\infty)$, are uniformly convex.

A Banach space $E$ is said to have a Fréchet differentiable norm if for all $x \in B=\{x \in E:\|x\|=1\}$

$$
\lim _{t \rightarrow 0} \frac{\|x+t \gamma\|-\|x\|}{t}
$$

exists and is attained uniformly in $y \in B$. It is well known that uniformly smooth Banach spaces has a Fréchet differentiable norm.

In order to prove our results, we need the following lemmas.

Lemma 2 (see [19]). Let $C$ be a nonempty close convex subset of a real Banach space $E$ which has the Fréchet differentiable norm. For $x \in E$, let $\rho$ be defined for $0<t<\infty$ by

$$
\rho(t)=\sup _{\gamma \in B}\left|\frac{\|x+t \gamma\|^{2}-\|x\|^{2}}{t}-2\langle\gamma, j(x)\rangle\right| .
$$

Then, $\lim _{t \rightarrow 0} \rho(t)=0$ and

$$
\|x+h\|^{2} \leq\|x\|^{2}+2\langle h, j(x)\rangle+\|h\| \rho(\|h\|), \quad \forall h \in E \backslash\{0\} .
$$


It is shown in [19] that if $E=H$, a real Hilbert space, then $\rho(t)=t$, for $t>0$. In our general setting, throughout this paper we assume that $\rho(t) \leq 2 t$.

Lemma 3. Let $E$ be a real Banach space. Then the following inequality holds:

$$
\begin{aligned}
& \|x+y\|^{2} \leq\|x\|^{2}+\langle y, j(x+y)\rangle, \\
& \forall x, y \in H, j(x+y) \in J(x+y) .
\end{aligned}
$$

Lemma 4 (see [20]). Let E be a uniformly convex Banach space and $B_{R}(0)$ a closed ball of $E$. Then, there exists a continuous strictly increasing convex function $g:[0, \infty) \rightarrow$ $[0, \infty)$ with $g(0)=0$ such that

$$
\begin{gathered}
\left\|\alpha_{0} x_{0}+\alpha_{1} x_{1}+\alpha_{2} x_{2}+\cdots+\alpha_{k} x_{k}\right\|^{2} \\
\leq \sum_{i=0}^{k} \alpha_{i}\left\|x_{i}\right\|^{2}-\alpha_{i} \alpha_{j} g\left(\left\|x_{i}-x_{j}\right\|\right),
\end{gathered}
$$

for each $\alpha_{i} \in(0,1)$ and for $x_{i} \in B_{R}(0):=\{x \in E:\|x\| \leq R\}$, $i=0,1,2, \ldots, k$ with $\sum_{i=0}^{k} \alpha_{i}=1$.

Lemma 5 (see [21]). Let $\left\{a_{n}\right\}$ be a sequence of nonnegative real numbers satisfying the following relation:

$$
a_{n+1} \leq\left(1-\alpha_{n}\right) a_{n}+\alpha_{n} \delta_{n}, \quad n \geq n_{0},
$$

where $\left\{\alpha_{n}\right\} \subset(0,1)$ and $\left\{\delta_{n}\right\} \subset R$ satisfying the following conditions: $\lim _{n \rightarrow \infty} \alpha_{n}=0, \sum_{n=1}^{\infty} \alpha_{n}=\infty$, and $\lim \sup _{n \rightarrow \infty} \delta_{n} \leq 0$. Then, $\lim _{n \rightarrow \infty} a_{n}=0$.

Lemma 6 (see [17]). Let $C$ be a nonempty closed convex subset of a real uniformly convex Banach space $E$ which has the Fréchet differentiable norm. Let $T: C \rightarrow C$ be an asymptotically $k$-strict pseudocontractive mapping with fixed point of $T, F(T):=\{x \in C: T x=x\} \neq \emptyset$. Then $(I-T)$ is demiclosed at zero, that is, if $x_{n} \rightarrow x$ and $T x_{n}-x_{n} \rightarrow 0$, as $n \rightarrow \infty$, then $x=T(x)$.

Lemma 7 (see [22]). Let $\left\{a_{n}\right\}$ be sequences of real numbers such that there exists a subsequence $\left\{n_{i}\right\}$ of $\{n\}$ such that $a_{n_{i}}<$ $a_{n_{i}+1}$ for all $i \in N$. Then there exists a nondecreasing sequence $\left\{m_{k}\right\} \subset N$ such that $m_{k} \rightarrow \infty$ and the following properties are satisfied by all (sufficiently large) numbers $k \in N$ :

$$
a_{m_{k}} \leq a_{m_{k}+1}, \quad a_{k} \leq a_{m_{k}+1} .
$$

In fact, $m_{k}=\max \left\{j \leq k: a_{j}<a_{j+1}\right\}$.

\section{Main Results}

We now prove our main theorem.

Theorem 8. Let $C$ be a nonempty, closed, and convex subset of a real uniformly convex Banach space E which has Fréchet differentiable norm possessing a weakly sequentially continuous duality mapping from $E$ into $E^{*}$. Let $T_{i}: C \rightarrow C$ be asymptotically $k_{i}$-strict pseudocontractive mappings for $0 \leq k_{i}<1$ with sequences $\left\{l_{n, i}\right\} \subset[1, \infty)$, for $i=1,2, \ldots, N$. Assume that $F:=\cap_{i=1}^{N} F\left(T_{i}\right)$ is nonempty. Let $\left\{x_{n}\right\}$ be a sequence defined by $x_{1}=u \in C$ and

$$
x_{n+1}=\alpha_{n} u+\left(1-\alpha_{n}\right)\left(\left(1-\beta_{n}\right) x_{n}+\beta_{n} S_{n} x_{n}\right), \quad n \geq 1,
$$

where $S_{n}:=\theta_{n, 1} T_{1}^{n}+\theta_{n, 2} T_{2}^{n}+\cdots+\theta_{n, N} T_{N}^{n}$, such that $\theta_{n, 1}+$ $\theta_{n, 2}+\cdots+\theta_{n, N}=1$, for each $n \geq 1,\left\{\alpha_{n}\right\},\left\{\theta_{n, i}\right\} \subset(0, c) \subset$ $(0,1)$, satisfying $\liminf \theta_{n, i}>0, \lim _{n \rightarrow \infty} \alpha_{n}=0, \sum \alpha_{n}=\infty$, $\lim _{n \rightarrow \infty}\left(\left(l_{n, i}-1\right) / \alpha_{n}\right)=0$, for $i=1,2, \ldots, N$ and $\left\{\beta_{n}\right\} \subset$ $[a, b] \subset(0, k)(a, b$, and $c$ constants $)$, for $k=\min _{1 \leq i \leq N}\left\{k_{i}\right\}$, Then the sequence $\left\{x_{n}\right\}$ generated by (14) converges strongly to a common fixed point of $\left\{T_{i}: i=1,2, \ldots, N\right\}$.

Proof. Fix $x^{*} \in F$. Let $y_{n}=\left(1-\beta_{n}\right) x_{n}+\beta_{n} S_{n} x_{n}$ and $l_{n}:=$ $\max \left\{l_{n, i}: i=1,2, \ldots, N\right\}$. Then, using Lemma 2 and (3) we have that

$$
\begin{aligned}
\| y_{n}- & x^{*} \|^{2} \\
= & \left\|\left(x_{n}-x^{*}\right)-\beta_{n}\left(x_{n}-S_{n} x_{n}\right)\right\|^{2} \\
= & \|\left(x_{n}-x^{*}\right) \\
& \quad-\beta_{n}\left(x_{n}-\left(\theta_{n, 1} T_{1}^{n}+\theta_{n, 2} T_{2}^{n}+\cdots+\theta_{n, N} T_{N}^{n}\right) x_{n}\right) \|^{2} \\
\leq & \left\|x_{n}-x^{*}\right\|^{2} \\
& -2 \beta_{n}\left\langle\theta_{n, 1}\left(x_{n}-T_{1}^{n} x_{n}\right)+\theta_{n, 2}\left(x_{n}-T_{2}^{n} x_{n}\right)\right. \\
& \left.\quad+\cdots+\theta_{n, N}\left(x_{n}-T_{N}^{n} x_{n}\right), j\left(x_{n}-x^{*}\right)\right\rangle \\
& +\beta_{n}\left\|x_{n}-S_{n} x_{n}\right\| \rho\left(\beta_{n}\left\|x_{n}-S_{n} x_{n}\right\|\right) \\
\leq & \left\|x_{n}-x^{*}\right\|^{2}-2 \beta_{n} \theta_{n, 1}\left\langle x_{n}-T_{1}^{n} x_{n}, j\left(x_{n}-x^{*}\right)\right\rangle \\
& -2 \beta_{n} \theta_{n, 2}\left\langle x_{n}-T_{2}^{n} x_{n}, j\left(x_{n}-x^{*}\right)\right\rangle \\
& -\cdots-2 \beta_{n} \theta_{n, N}\left\langle x_{n}-T_{N}^{n} x_{n}, j\left(x_{n}-x^{*}\right)\right\rangle \\
& +2 \beta_{n}^{2}\left\|x_{n}-S_{n} x_{n}\right\|^{2} \\
\leq & \left\|x_{n}-x^{*}\right\|^{2} \\
& -2 \beta_{n} \theta_{n, 1}\left[k\left\|x_{n}-T_{1}^{n} x_{n}\right\|^{2}-\left(l_{n}-1\right)\left\|x_{n}-x^{*}\right\|^{2}\right] \\
& -2 \beta_{n} \theta_{n, 2}\left[k\left\|x_{n}-T_{2}^{n} x_{n}\right\|^{2}-\left(l_{n}-1\right)\left\|x_{n}-x^{*}\right\|^{2}\right] \\
& -\cdots-2 \beta_{n} \theta_{n, N}\left[k\left\|x_{n}-T_{N}^{n} x_{n}\right\|^{2}-\left(l_{n}-1\right)\left\|x_{n}-x^{*}\right\|^{2}\right] \\
& +2 \beta_{n}^{2}\left\|x_{n}-S_{n} x_{n}\right\|^{2} \\
\leq & {\left[1+2 \beta_{n}\left(l_{n}-1\right)\right]\left\|x_{n}-x^{*}\right\|^{2}-2 \beta_{n} \theta_{n, 1} k\left\|x_{n}-T_{1}^{n} x_{n}\right\|^{2} } \\
& -2 \beta_{n} \theta_{n, 2} k\left\|x_{n}-T_{2}^{n} x_{n}\right\|^{2} \\
& -\cdots-2 \beta_{n} \theta_{n, N} k\left\|x_{n}-T_{N}^{n} x_{n}\right\|^{2} \\
& +2 \beta_{n}^{2}\left\|x_{n}-S_{n} x_{n}\right\|^{2} . \\
&
\end{aligned}
$$


On the other hand using Lemma 4 we get that

$$
\begin{aligned}
\left\|x_{n}-S_{n} x_{n}\right\|^{2}= & \left\|x_{n}-\left(\theta_{n, 1} T_{1}^{n}+\theta_{n, 2} T_{2}^{n}+\cdots+\theta_{n, N} T_{N}^{n}\right) x_{n}\right\|^{2} \\
= & \| \theta_{n, 1}\left(x_{n}-T_{1}^{n} x_{n}\right)+\theta_{n, 2}\left(x_{n}-T_{2}^{n} x_{n}\right) \\
& +\cdots+\theta_{n, N}\left(x_{n}-T_{N}^{n} x_{n}\right) \|^{2} \\
\leq & \theta_{n, 1}\left\|x_{n}-T_{1}^{n} x_{n}\right\|^{2}+\theta_{n, 2}\left\|x_{n}-T_{2}^{n} x_{n}\right\|^{2} \\
& +\cdots+\theta_{n, N}\left\|x_{n}-T_{N}^{n} x_{n}\right\|^{2} .
\end{aligned}
$$

Now substituting (16) into (15) we obtain that

$$
\begin{aligned}
\left\|y_{n}-x^{*}\right\|^{2} \leq & {\left[1+2 \beta_{n}\left(l_{n}-1\right)\right]\left\|x_{n}-x^{*}\right\|^{2} } \\
& -2 \beta_{n} \theta_{n, 1}\left(k-\beta_{n}\right)\left\|x_{n}-T_{1}^{n} x_{n}\right\|^{2} \\
& -2 \beta_{n} \theta_{n, 2}\left(k-\beta_{n}\right)\left\|x_{n}-T_{2}^{n} x_{n}\right\|^{2} \\
& -\cdots-2 \beta_{n} \theta_{n, N}\left(k-\beta_{n}\right)\left\|x_{n}-T_{N}^{n} x_{n}\right\|^{2} \\
\leq & {\left[1+2 \beta_{n}\left(l_{n}-1\right)\right]\left\|x_{n}-x^{*}\right\|^{2}, }
\end{aligned}
$$

since $\left(k-\beta_{n}\right) \geq 0$ for each $n \geq 1$. Then now, from (14) and (18) we get that

$$
\begin{aligned}
\left\|x_{n+1}-x^{*}\right\|^{2}= & \left\|\alpha_{n}\left(u-x^{*}\right)+\left(1-\alpha_{n}\right)\left(y_{n}-x^{*}\right)\right\|^{2} \\
\leq & \alpha_{n}\left\|u-x^{*}\right\|^{2}+\left(1-\alpha_{n}\right)\left\|y_{n}-x^{*}\right\|^{2} \\
\leq & \alpha_{n}\left\|u-x^{*}\right\|^{2} \\
& +\left(1-\alpha_{n}\right)\left[1+2 \beta_{n}\left(l_{n}-1\right)\left\|x_{n}-x^{*}\right\|^{2}\right] \\
\leq & \alpha_{n}\left\|u-x^{*}\right\|^{2} \\
& +\left(1-\alpha_{n}+\epsilon \alpha_{n}\right)\left\|x_{n}-x^{*}\right\|^{2}, \quad \forall n \geq N_{0}, \\
\leq & \alpha_{n}\left\|u-x^{*}\right\|^{2} \\
& +\left(1-\alpha_{n}(1-\epsilon)\right)\left\|x_{n}-x^{*}\right\|^{2}, \quad \forall n \geq N_{0},
\end{aligned}
$$

where $N_{0}$ is a positive integer such that $2\left(1-\alpha_{n}\right) \beta_{n}\left(l_{n}-\right.$ $1) / \alpha_{n}<\epsilon$, for all $n \geq N_{0}$, for some $\epsilon>0$. Therefore, by induction,

$$
\begin{array}{r}
\left\|x_{n+1}-x^{*}\right\|^{2} \leq \max \left\{\left\|x_{N_{0}}-x^{*}\right\|^{2},(1-\epsilon)^{-1}\left\|u-x^{*}\right\|^{2}\right\}, \\
\forall n \geq N_{0},
\end{array}
$$

which implies that $\left\{x_{n}\right\}$ and hence $\left\{y_{n}\right\}$ are bounded.
Furthermore, from (14), Lemma 3, and (17) we get that

$$
\begin{aligned}
\| x_{n+1} & -x^{*} \|^{2} \\
= & \left\|\alpha_{n}\left(u-x^{*}\right)+\left(1-\alpha_{n}\right)\left(y_{n}-x^{*}\right)\right\|^{2} \\
\leq & \left(1-\alpha_{n}\right)\left\|y_{n}-x^{*}\right\|^{2} \\
& +2 \alpha_{n}\left\langle u-x^{*}, J\left(x_{n+1}-x^{*}\right)\right\rangle \\
\leq & \left(1-\alpha_{n}\right)\left[1+2 \beta_{n}\left(l_{n}-1\right)\right]\left\|x_{n}-x^{*}\right\|^{2} \\
& +2 \alpha_{n}\left\langle u-x^{*}, J\left(x_{n+1}-x^{*}\right)\right\rangle \\
& -2 \beta_{n} \theta_{n, 1}\left(k-\beta_{n}\right)\left(1-\alpha_{n}\right)\left\|x_{n}-T_{1}^{n} x_{n}\right\|^{2} \\
& -2 \beta_{n} \theta_{n, 2}\left(k-\beta_{n}\right)\left(1-\alpha_{n}\right)\left\|x_{n}-T_{2}^{n} x_{n}\right\|^{2} \\
& -\cdots-2 \beta_{n} \theta_{n, N}\left(k-\beta_{n}\right)\left(1-\alpha_{n}\right)\left\|x_{n}-T_{N}^{n} x_{n}\right\|^{2} \\
\leq & \left(1-\alpha_{n}\right)\left\|x_{n}-x^{*}\right\|^{2}+2 \alpha_{n}\left\langle u-x^{*}, J\left(x_{n+1}-x^{*}\right)\right\rangle \\
& +2 \beta_{n} M\left(l_{n}-1\right) \\
& -2 \beta_{n} \theta_{n, 1}\left(1-\alpha_{n}\right)\left(k-\beta_{n}\right)\left\|x_{n}-T_{1}^{n} x_{n}\right\|^{2} \\
& -2 \beta_{n} \theta_{n, 2}\left(k-\beta_{n}\right)\left(1-\alpha_{n}\right)\left\|x_{n}-T_{2}^{n} x_{n}\right\|^{2} \\
& -\cdots-2 \beta_{n} \theta_{n, N}\left(k-\beta_{n}\right)\left(1-\alpha_{n}\right)\left\|x_{n}-T_{N}^{n} x_{n}\right\|^{2}
\end{aligned}
$$

$$
\begin{aligned}
\leq & \left(1-\alpha_{n}\right)\left\|x_{n}-x^{*}\right\|^{2}+2 \alpha_{n}\left\langle u-x^{*}, J\left(x_{n+1}-x^{*}\right)\right\rangle \\
& +2 \beta_{n} M\left(l_{n}-1\right),
\end{aligned}
$$

for some $M>0$.

Now, the rest of the proof is divided into two parts.

Case 1. Suppose that there exists $N_{1} \geq 0$ such that $\left\{\left\|x_{n}-x^{*}\right\|\right\}$ is decreasing for all $n \geq N_{1}$. Then we have that $\left.\left\{\left\|x_{n}-x^{*}\right\|\right)\right\}$ is convergent. Then from (21) and the assumptions on $\left\{\beta_{n}\right\},\left\{\alpha_{n}\right\}$, and $\left\{l_{n}\right\}$ we have that $\beta_{n} \theta_{n, i}(1-c)\left(k-\beta_{n}\right)\left\|x_{n}-T_{i}^{n} x_{n}\right\|^{2} \rightarrow 0$, as $n \rightarrow \infty$, which implies that

$$
x_{n}-T_{i}^{n} x_{n} \longrightarrow 0, \quad \text { as } n \longrightarrow \infty \text {, }
$$

for $i=1,2, \ldots, N$. Then from (14) we obtain that

$$
x_{n+1}-y_{n}=\alpha_{n}\left(u-y_{n}\right) \longrightarrow 0, \quad \text { as } n \longrightarrow \infty .
$$

Again, from (23) we get that

$$
\begin{aligned}
\left\|y_{n}-x_{n}\right\|= & \left\|\beta_{n}\left(S_{n} x_{n}-x_{n}\right)\right\| \leq\left\|S_{n} x_{n}-x_{n}\right\| \\
\leq & \theta_{n, 1}\left\|T_{1}^{n} x_{n}-x_{n}\right\|+\theta_{n, 2}\left\|T_{2}^{n} x_{n}-x_{n}\right\| \\
& +\cdots+\theta_{n, N}\left\|T_{N}^{n} x_{n}-x_{n}\right\| \longrightarrow 0,
\end{aligned}
$$

as $n \rightarrow \infty$. Thus, (24) and (25) imply that

$$
x_{n+1}-x_{n} \longrightarrow 0, \quad \text { as } n \longrightarrow \infty \text {. }
$$


Therefore, since each $T_{i}$, for $i=1,2, \ldots, N$, is uniformly $L$ Lipschitzian and

$$
\begin{aligned}
\| x_{n}- & T_{i} x_{n} \| \\
\leq & \left\|x_{n}-x_{n+1}\right\| \\
& +\left\|x_{n+1}-T_{i}^{n+1} x_{n+1}\right\|+\left\|T_{i}^{n+1} x_{n+1}-T_{i}^{n+1} x_{n}\right\| \\
& +\left\|T_{i}^{n+1} x_{n}-T_{i} x_{n}\right\| \\
\leq & \left\|x_{n}-x_{n+1}\right\|+\left\|x_{n+1}-T_{i}^{n+1} x_{n+1}\right\|+L\left\|x_{n+1}-x_{n}\right\| \\
& +\left\|T_{i}\left(T_{i}^{n} x_{n}\right)-T_{i} x_{n}\right\| \\
= & (1+L)\left\|x_{n}-x_{n+1}\right\|+\left\|x_{n+1}-T_{i}^{n+1} x_{n+1}\right\| \\
& +\left\|T_{i}\left(T_{i}^{n} x_{n}\right)-T_{i} x_{n}\right\|,
\end{aligned}
$$

we have from (23), (26), and uniform continuity of $T_{i}$ that

$$
\left\|x_{n}-T_{i} x_{n}\right\| \longrightarrow 0, \quad \text { as } n \longrightarrow \infty,
$$

for each $i=1,2, \ldots, N$. Furthermore, the fact that $\left\{x_{n}\right\}$ is bounded and $E$ is reflexive implies that we can choose a subsequence $\left\{x_{n_{i}+1}\right\}$ of $\left\{x_{n+1}\right\}$ such that $x_{n_{i}+1} \rightarrow z$ and

$$
\begin{aligned}
\limsup _{n \rightarrow \infty} & \left\langle u-x^{*}, J\left(x_{n+1}-x^{*}\right)\right\rangle \\
& =\lim _{i \rightarrow \infty}\left\langle u-x^{*}, J\left(x_{n_{i}+1}-x^{*}\right)\right\rangle .
\end{aligned}
$$

Now, from (26) we get that $x_{n_{i}} \rightarrow z$ and from Lemma 6 we have that $z \in F\left(T_{i}\right)$, for each $i=1,2, \ldots, N$. Hence, $z \in \cap_{i=1}^{N} F\left(T_{i}\right)$. Therefore, putting $x^{*}=z$ in (29) and using the fact that $J$ is weakly sequentially continuous we immediately obtain that $\lim \sup _{n \rightarrow \infty}\left\langle u-z, J\left(x_{n+1}-z\right)\right\rangle=\lim _{i \rightarrow \infty}\langle u-$ $\left.z, J\left(x_{n_{i}+1}-z\right)\right\rangle=\langle u-z, J(z-z)\rangle=0$. Again, putting $x^{*}=z$ in inequality (22), we get that

$$
\begin{aligned}
\left\|x_{n+1}-z\right\|^{2} \leq & \left(1-\alpha_{n}\right)\left\|x_{n}-z\right\|^{2} \\
& +2 \alpha_{n}\left\langle u-z, J\left(x_{n+1}-z\right)\right\rangle \\
& +2 \beta_{n} M\left(l_{n}-1\right),
\end{aligned}
$$

and, hence, it follows from (30) and Lemma 5 that $\left\|x_{n}-z\right\| \rightarrow$ 0 , as $n \rightarrow \infty$. Consequently, $x_{n} \rightarrow z$.

Case 2. Suppose that there exists a subsequence $\left\{n_{i}\right\}$ of $\{n\}$ such that

$$
\left\|x_{n_{i}}-x^{*}\right\|<\left\|x_{n_{i}+1}-x^{*}\right\|
$$

for all $i \in N$. Then, by Lemma 7, there exists a nondecreasing sequence $\left\{m_{j}\right\} \subset N$ such that $m_{j} \rightarrow \infty,\left\|x_{m_{j}}-x^{*}\right\| \leq$ $\left\|x_{m_{j}+1}-x^{*}\right\|$ and $\left\|x_{j}-x^{*}\right\| \leq\left\|x_{m_{j}+1}-x^{*}\right\|$ for all $j \in N$. Then from (21) and following the method of Case 1, we get that

$$
\left\|x_{m_{j}}-T_{i}^{m_{j}} x_{m_{j}}\right\| \longrightarrow 0, \quad \text { as } j \longrightarrow \infty
$$

for each $i=1,2, \ldots, N$. Thus, again following the method of Case 1 , we obtain that $x_{m_{j}+1}-x_{m_{j}} \rightarrow 0$ and $x_{m_{j}}-T_{i} x_{m_{j}} \rightarrow 0$, as $j \rightarrow \infty$, for each $i=1,2, \ldots, N$ and there exists $z^{*} \epsilon$ $\cap_{i=1}^{N} F\left(T_{i}\right)$ such that

$$
\limsup _{j \rightarrow \infty}\left\langle u-z^{*}, J\left(x_{m_{j}+1}-z^{*}\right)\right\rangle=0
$$

Then now, putting $x^{*}=z^{*}$ in (22) we have that

$$
\begin{aligned}
\left\|x_{m_{j}+1}-z^{*}\right\|^{2} \leq & \left(1-\alpha_{m_{j}}\right)\left\|x_{m_{j}}-z^{*}\right\|^{2} \\
& +2 \alpha_{m_{j}}\left\langle u-z^{*}, J\left(x_{m_{j}+1}-z^{*}\right)\right\rangle \\
& +2 \beta_{m_{j}} M\left(l_{m_{j}}-1\right) .
\end{aligned}
$$

Since $\left\|x_{m_{j}}-z^{*}\right\|^{2} \leq\left\|x_{m_{j}+1}-z^{*}\right\|^{2}$, (34) implies that

$$
\begin{aligned}
\alpha_{m_{j}}\left\|x_{m_{j}}-z^{*}\right\|^{2} \leq & \left\|x_{m_{j}}-z^{*}\right\|^{2}-\left\|x_{m_{j}+1}-z^{*}\right\|^{2} \\
& +2 \alpha_{m_{j}}\left\langle u-z^{*}, J\left(x_{m_{j}+1}-z^{*}\right)\right\rangle \\
& +2 \beta_{m_{j}} M\left(l_{m_{j}}-1\right) .
\end{aligned}
$$

Moreover, since $\alpha_{m_{j}}>0$, inequality (35) gives that

$$
\begin{gathered}
\left\|x_{m_{j}}-z^{*}\right\|^{2} \leq 2\left\langle u-z^{*}, J\left(x_{m_{j}+1}-z^{*}\right)\right\rangle \\
+\frac{2 \beta_{m_{j}} M\left(l_{m_{j}}-1\right)}{\alpha_{m_{j}}} .
\end{gathered}
$$

Then, from (33) and the fact that $2 \beta_{m_{j}} M\left(l_{m_{j}}-1\right) / \alpha_{m_{j}} \rightarrow 0$, we obtain that $\left\|x_{m_{j}}-z^{*}\right\| \rightarrow 0$, as $j \rightarrow \infty$. This together with (34) gives that $\left\|x_{m_{j}+1}-z^{*}\right\| \rightarrow 0$, as $j \rightarrow \infty$. But $\left\|x_{j}-z^{*}\right\| \leq\left\|x_{m_{j}+1}-z^{*}\right\|$, for all $j \in N$; thus we obtain that $x_{j} \rightarrow z^{*}$. Therefore, from the above two cases, we can conclude that $\left\{x_{n}\right\}$ converges strongly to an element of $F$ and the proof is complete.

If, in Theorem 8, we assume a single asymptotically $k$-strict pseudocontractive mapping we get the following corollary.

Corollary 9. Let $C$ be a nonempty, closed, and convex subset of a real uniformly convex Banach space $E$ which has Fréchet differentiable norm possessing a weakly sequentially continuous duality mapping from $E$ into $E^{*}$. Let $T: C \rightarrow C$ be an asymptotically $k$-strict pseudocontractive mapping for $0 \leq k<$ 1 with sequences $\left\{l_{n}\right\} \subset[1, \infty)$. Assume that $F(T)$ is nonempty. Let $\left\{x_{n}\right\}$ be a sequence defined by $x_{1}=u \in C$ and

$$
x_{n+1}=\alpha_{n} u+\left(1-\alpha_{n}\right)\left(\left(1-\beta_{n}\right) x_{n}+\beta_{n} T^{n} x_{n}\right), \quad n \geq 1 \text {, }
$$

where $\left\{\alpha_{n}\right\} \subset(0, c) \subset(0,1)$, satisfying $\lim _{n \rightarrow \infty} \alpha_{n}=0, \sum \alpha_{n}=$ $\infty, \lim _{n \rightarrow \infty}\left(\left(l_{n}-1\right) / \alpha_{n}\right)=0$, and $\left\{\beta_{n}\right\} \subset[a, b] \subset(0, k)(a$, $b$, and $c$ constants). Then the sequence $\left\{x_{n}\right\}$ generated by (37) converges strongly to a fixed point of $T$. 
Proof. Putting $T=T_{1}=T_{2}=\cdots=T_{N}$ in (14), we get that $S_{n}=T^{n}$ and the scheme reduces to scheme (37) and following the method of proof of Theorem 8 we get that (see (21) and (22))

$$
\begin{aligned}
\left\|x_{n+1}-x^{*}\right\|^{2} \leq & \left(1-\alpha_{n}\right)\left\|x_{n}-x^{*}\right\|^{2} \\
& +2 \alpha_{n}\left\langle u-x^{*}, J\left(x_{n+1}-x^{*}\right)\right\rangle \\
& -2 \beta_{n}\left(k-\beta_{n}\right)\left(1-\alpha_{n}\right)\left\|x_{n}-T^{n} x_{n}\right\|^{2} \\
& +2 \beta_{n} M^{\prime}\left(l_{n}-1\right) \\
\leq & \left(1-\alpha_{n}\right)\left\|x_{n}-x^{*}\right\|^{2} \\
& +2 \alpha_{n}\left\langle u-x^{*}, J\left(x_{n+1}-x^{*}\right)\right\rangle \\
& -2 \beta_{n}\left(k-\beta_{n}\right)(1-c)\left\|x_{n}-T^{n} x_{n}\right\|^{2} \\
& +2 \beta_{n} M^{\prime}\left(l_{n}-1\right) \\
\leq & \left(1-\alpha_{n}\right)\left\|x_{n}-x^{*}\right\|^{2} \\
& +2 \alpha_{n}\left\langle u-x^{*}, J\left(x_{n+1}-x^{*}\right)\right\rangle \\
& +2 \beta_{n} M^{\prime}\left(l_{n}-1\right)
\end{aligned}
$$

for some $M^{\prime}>0$. Now, considering cases, as in the proof of Theorem 8 , we obtain the required result.

Corollary 10. Let $K$ be a nonempty, closed, and convex subset of $l_{p}, 1<p<\infty$. Let $T_{i}: C \rightarrow C$ be asymptotically $k_{i}$-strict pseudocontractive mappings for $0 \leq k_{i}<1$ with sequences $\left\{l_{n, i}\right\} \subset[1, \infty)$, for $i=1,2, \ldots, N$. Assume that $F:=\cap_{i=1}^{n} F\left(T_{i}\right)$ is nonempty. Let $\left\{x_{n}\right\}$ be a sequence defined by $x_{1}=u \in C$ and

$$
x_{n+1}=\alpha_{n} u+\left(1-\alpha_{n}\right)\left(\left(1-\beta_{n}\right) x_{n}+\beta_{n} S_{n} x_{n}\right), \quad n \geq 1 \text {, }
$$

where $S_{n}:=\theta_{n, 1} T_{1}^{n}+\theta_{n, 2} T_{2}^{n}+\cdots+\theta_{n, N} T_{N}^{n}$, such that $\theta_{n, 1}+$ $\theta_{n, 2}+\cdots+\theta_{n, N}=1$, for each $n \geq 1,\left\{\alpha_{n}\right\},\left\{\theta_{n, i}\right\} \subset(0, c) \subset(0,1)$, satisfying $\liminf _{n \rightarrow \infty} \theta_{n, i}>0, \lim _{n \rightarrow \infty} \alpha_{n}=0, \sum \alpha_{n}=\infty$, $\lim _{n \rightarrow \infty}\left(\left(l_{n, i}-1\right) / \alpha_{n}\right)=0$ and $\left\{\beta_{n}\right\} \subset[a, b] \subset(0, k)$ (for $a, b$, and c constants), for $k=\min _{1 \leq i \leq N}\left\{k_{i}\right\}$. Then the sequence $\left\{x_{n}\right\}$ generated by (39) converges strongly to a common fixed point of $\left\{T_{i}: i=1,2, \ldots, N\right\}$.

Proof. We note that $l_{p}, 1<p<\infty$, spaces are uniformly convex which have Fréchet differentiable norm possessing a weakly sequentially continuous duality mapping from $E$ into $E^{*}$ (see, e.g., [18]). Thus, the result follows from Theorem 8 .

Corollary 11. Let $K$ be a nonempty, closed, and convex subset of $l_{p}, 1<p<\infty$. Let $T: C \rightarrow C$ be an asymptotically $k$-strict pseudocontractive mapping for some $0 \leq k<1$ with sequences $\left\{l_{n}\right\} \subset[1, \infty)$. Assume that $F(T)$ is nonempty. Let $\left\{x_{n}\right\}$ be a sequence defined by $x_{1}=u \in C$ and

$$
x_{n+1}=\alpha_{n} u+\left(1-\alpha_{n}\right)\left(\left(1-\beta_{n}\right) x_{n}+\beta_{n} T^{n} x_{n}\right), \quad n \geq 1,
$$

where $\left\{\alpha_{n}\right\} \subset(0, c) \subset(0,1)$, and $\left\{\beta_{n}\right\} \subset[a, b] \subset(0, k)$ (for $a$, $b$, and $c$ constants) satisfying $\lim _{n \rightarrow \infty} \alpha_{n}=0, \sum \alpha_{n}=\infty$ and $\lim _{n \rightarrow \infty}\left(\left(l_{n}-1\right) / \alpha_{n}\right)=0$. Then the sequence $\left\{x_{n}\right\}$ converges strongly to a fixed point of $T$.

If in Theorem 8 we have that $E=H$, a real Hilbert space, then $E$ is uniformly convex with Fréchet differentiable norm possessing a weakly sequentially continuous duality mapping. Thus, we have the following corollary.

Corollary 12. Let $C$ be a nonempty, closed, and convex subset of a real Hilbert space $H$. Let $T_{i}: C \rightarrow C$ be asymptotically $k_{i}$-strict pseudocontractive mappings for $0 \leq k_{i}<1$ with sequences $\left\{l_{n, i}\right\} \subset[1, \infty)$, for $i=1,2, \ldots, N$. Assume that $F:=\cap_{i=1}^{n} F\left(T_{i}\right)$ is nonempty. Let $\left\{x_{n}\right\}$ be a sequence defined by $x_{1}=u \in C$ and

$$
x_{n+1}=\alpha_{n} u+\left(1-\alpha_{n}\right)\left(\left(1-\beta_{n}\right) x_{n}+\beta_{n} S_{n} x_{n}\right), \quad n \geq 1,
$$

where $S_{n}:=\theta_{n, 1} T_{1}^{n}+\theta_{n, 2} T_{2}^{n}+\cdots+\theta_{n, N} T_{N}^{n}$, such that $\theta_{n, 1}+$ $\theta_{n, 2}+\cdots+\theta_{n, N}=1$, for each $n \geq 1,\left\{\alpha_{n}\right\},\left\{\theta_{n, i}\right\} \subset(0, c) \subset$ $(0,1)$, satisfying $\liminf _{n} \theta_{n, i}>0, \lim _{n \rightarrow \infty} \alpha_{n}=0, \sum \alpha_{n}=\infty$, $\lim _{n \rightarrow \infty}\left(\left(l_{n, i}-1\right) / \alpha_{n}\right)=0$ and $\left\{\beta_{n}\right\} \subset[a, b] \subset(0, k)$ (for $a, b$, and c constants), for $k=\min _{1 \leq i \leq N}\left\{k_{i}\right\}$. Then the sequence $\left\{x_{n}\right\}$ generated by (41) converges strongly to a common fixed point of $\left\{T_{i}: i=1,2, \ldots, N\right\}$.

Corollary 13. Let $C$ be a nonempty, closed, and convex subset of a real Hilbert space $H$. Let $T: C \rightarrow C$ be an asymptotically $k$-strict pseudocontractive mapping for some $0 \leq k<1$ with sequences $\left\{l_{n}\right\} \subset[1, \infty)$. Assume that $F(T)$ is nonempty. Let $\left\{x_{n}\right\}$ be a sequence defined by $x_{1}=u \in C$ and

$$
x_{n+1}=\alpha_{n} u+\left(1-\alpha_{n}\right)\left(\left(1-\beta_{n}\right) x_{n}+\beta_{n} T^{n} x_{n}\right), \quad n \geq 1,
$$

where $\left\{\alpha_{n}\right\} \subset(0, c) \subset(0,1)$, and $\left\{\beta_{n}\right\} \subset[a, b] \subset(0, k)$ (for $a$, $b$, and $c$ constants) satisfying $\lim _{n \rightarrow \infty} \alpha_{n}=0, \sum \alpha_{n}=\infty$ and $\lim _{n \rightarrow \infty}\left(\left(l_{n}-1\right) / \alpha_{n}\right)=0$. Then the sequence $\left\{x_{n}\right\}$ converges strongly to a fixed point of $T$.

Remark 14. We note that Corollary 9 generalizes several recent results of this nature. Particularly, it extends Theorem KX of [12], Theorem 2 of Liu [5], and corresponding theorem of Schu [7] in the sense that our convergence is strong in more general Banach spaces possessing weakly sequentially continuous duality mappings without the requirement that $T$ be completely continuous.

Remark 15. Corollary 9 is an improvement of Theorem 3.2 of Osilike et al. [13] and Theorems 3.1 and 3.2 of Zhang and Xie [17] in the sense that our convergence is strong without the requirement that $\liminf _{n \rightarrow \infty} d\left(x_{n}, F(T)\right)=0$, provided that $E$ possesses weakly sequentially continuous duality mappings. 


\section{Acknowledgments}

N. Shahzad gratefully acknowledges research support from the Deanship of Scientific Research (DSR), King Abdulaziz University, Jeddah, Saudi Arabia.

\section{References}

[1] J. M. A. Toledano, T. D. Benavides, and G. L. Acedo, Measure of Noncoin Metric Fixed Point Theory, Birkhäauser, Boston, Mass, USA, 1997.

[2] F. E. Browder, "Convergence theorems for sequences of nonlinear operators in Banach spaces," Mathematische Zeitschrift, vol. 100, pp. 201-225, 1967.

[3] M. O. Osilike, "Iterative approximation of fixed points of asymptotically demicontractive mappings," Indian Journal of Pure and Applied Mathematics, vol. 29, no. 12, pp. 1291-1300, 1998.

[4] M. O. Osilike, S. C. Aniagbosor, and B. G. Akuchu, "Fixed points of asymptotically demicontractive mappings in arbitrary Banach spaces," Panamerican Mathematical Journal, vol. 12, no. 2, pp. 77-88, 2002.

[5] Q. Liu, "Convergence theorems of the sequence of iterates for asymptotically demicontractive and hemicontractive mappings," Nonlinear Analysis: Theory, Methods \& Applications, vol. 26, no. 11, pp. 1835-1842, 1996.

[6] X. Qin, S. Y. Cho, and J. K. Kim, "Convergence theorems on asymptotically pseudocontractive mappings in the intermediate sense," Fixed Point Theory and Applications, vol. 2010, Article ID 186874, 14 pages, 2010.

[7] J. Schu, "Iterative construction of fixed points of asymptotically nonexpansive mappings," Journal of Mathematical Analysis and Applications, vol. 158, no. 2, pp. 407-413, 1991.

[8] J. Schu, "Weak and strong convergence to fixed points of asymptotically nonexpansive mappings," Bulletin of the Australian Mathematical Society, vol. 43, no. 1, pp. 153-159, 1991.

[9] F. E. Browder and W. V. Petryshyn, "Construction of fixed points of nonlinear mappings in Hilbert space," Journal of Mathematical Analysis and Applications, vol. 20, pp. 197-228, 1967.

[10] T. L. Hicks and J. D. Kubicek, "On the Mann iteration process in a Hilbert space," Journal of Mathematical Analysis and Applications, vol. 59, no. 3, pp. 498-504, 1977.

[11] Z. Huang, "Mann and Ishikawa iterations with errors for asymptotically nonexpansive mappings," Computers \& Mathematics with Applications, vol. 37, no. 3, pp. 1-7, 1999.

[12] T.-H. Kim and H.-K. Xu, "Convergence of the modified Mann's iteration method for asymptotically strict pseudo-contractions," Nonlinear Analysis: Theory, Methods \& Applications, vol. 68, no. 9, pp. 2828-2836, 2008.

[13] M. O. Osilike, A. Udomene, D. I. Igbokwe, and B. G. Akuchu, "Demiclosedness principle and convergence theorems for $k$ strictly asymptotically pseudocontractive maps," Journal of Mathematical Analysis and Applications, vol. 326, no. 2, pp. 1334-1345, 2007.

[14] B. E. Rhoades, "Fixed point iterations using infinite matrices," Transactions of the American Mathematical Society, vol. 196, pp. 161-176, 1974.

[15] K.-K. Tan and H. K. Xu, "Fixed point iteration processes for asymptotically nonexpansive mappings," Proceedings of the American Mathematical Society, vol. 122, no. 3, pp. 733-739, 1994.
[16] H. Zegeye, M. Robdera, and B. Choudhary, "Convergence theorems for asymptotically pseudocontractive mappings in the intermediate sense," Computers \& Mathematics with Applications, vol. 62, no. 1, pp. 326-332, 2011.

[17] Y. Zhang and Z. Xie, “Convergence of modified Mann's iterative method for asymptotically $k$-strictly pseudocontractive mappings," Fixed Point Theory and Applications, vol. 2011, p. 100, 2011.

[18] W. Takahashi, Nonlinear Functional Analysis: Fixed Point Theory and Its Applications, Yokohama Publishers, Yokohama, Japan, 2000.

[19] P. Cholamjiak and S. Suantai, "Weak convergence theorems for a countable family of strict pseudocontractions in Banach spaces," Fixed Point Theory and Applications, vol. 2010, Article ID 632137, 2010.

[20] H. Zegeye and N. Shahzad, “Convergence of Mann's type iteration method for Generalized asymptotically nonexpansive mappings," Computers \& Mathematics with Applications, vol. 62, no. 11, pp. 4007-4014, 2011.

[21] H.-K. Xu, "Another control condition in an iterative method for nonexpansive mappings," Bulletin of the Australian Mathematical Society, vol. 65, no. 1, pp. 109-113, 2002.

[22] P.-E. Maingé, "Strong convergence of projected subgradient methods for nonsmooth and nonstrictly convex minimization," Set-Valued Analysis, vol. 16, no. 7-8, pp. 899-912, 2008. 


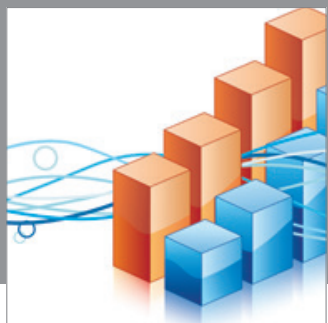

Advances in

Operations Research

mansans

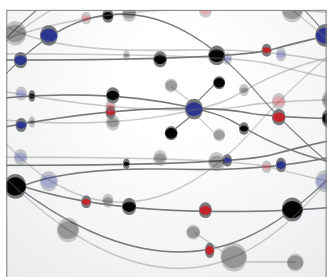

The Scientific World Journal
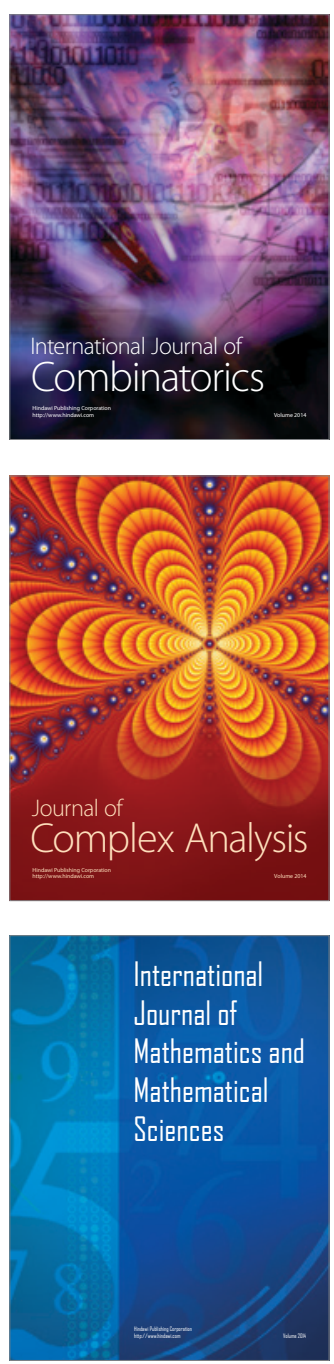
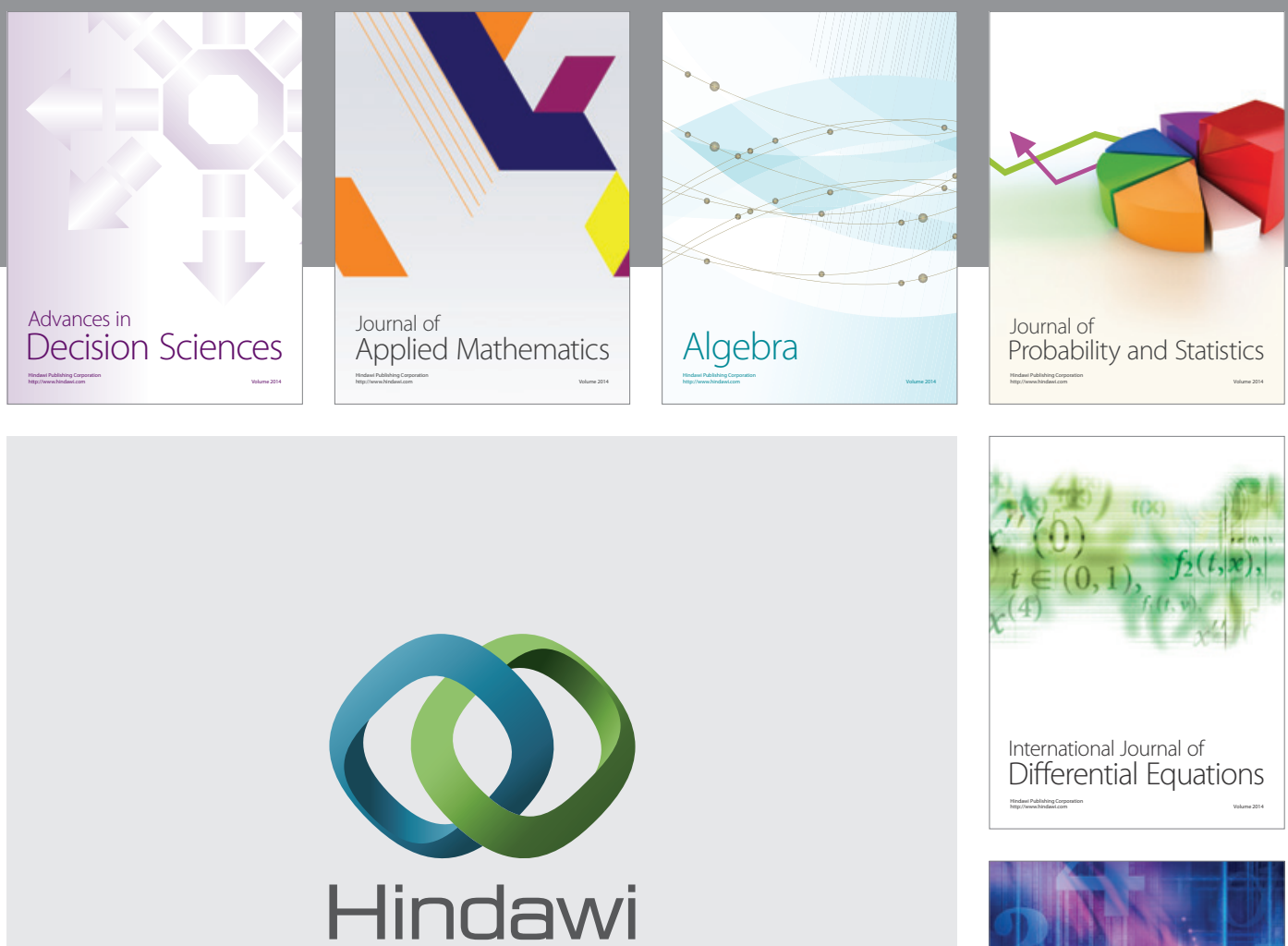

Submit your manuscripts at http://www.hindawi.com
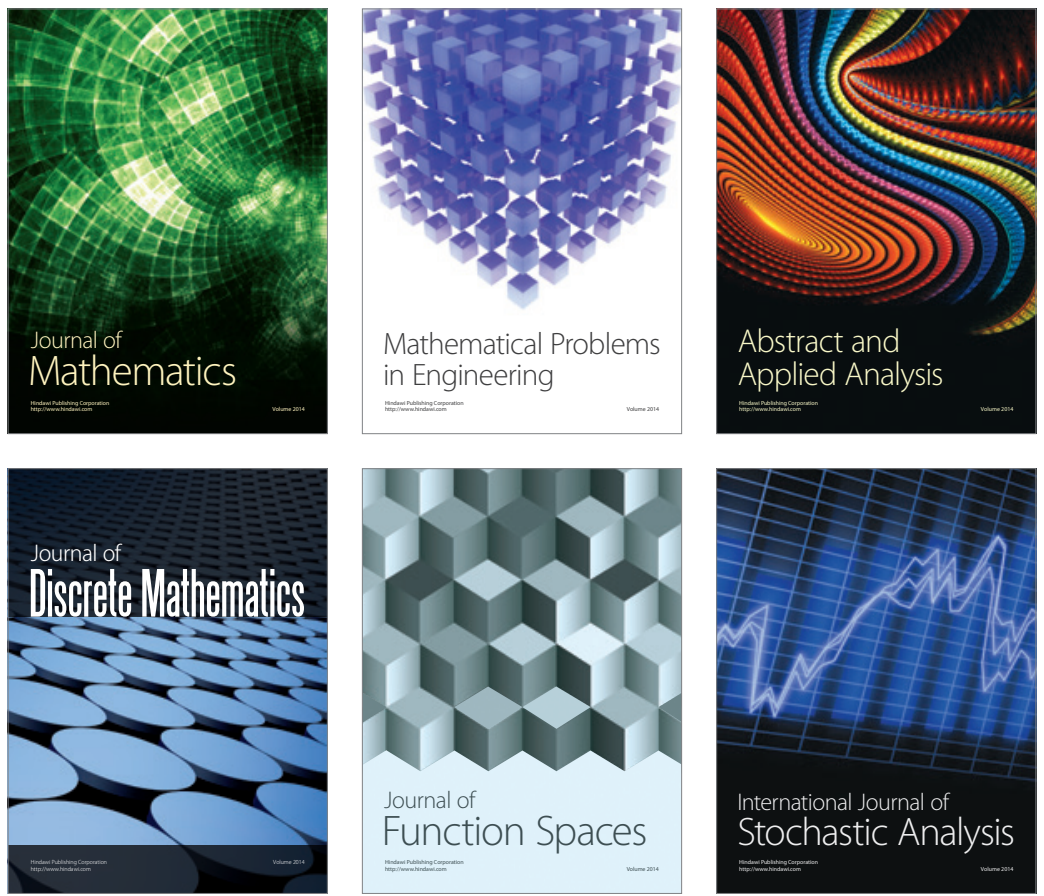

Journal of

Function Spaces

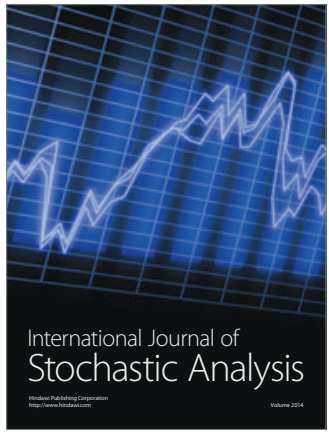

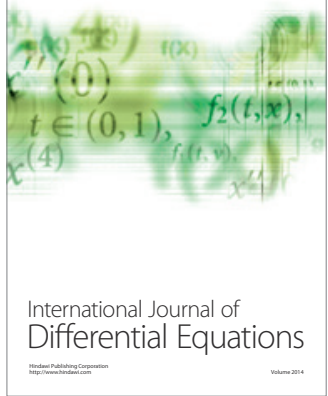
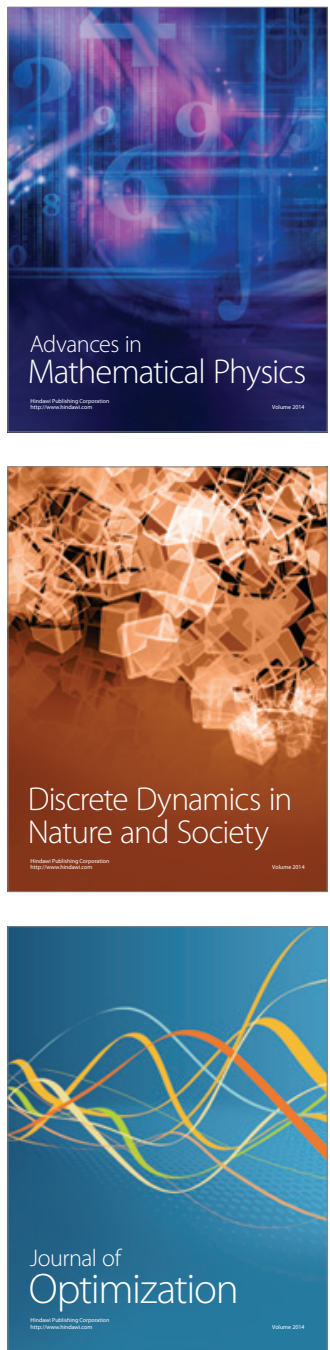